\title{
A Novel Fog Computing Approach for Minimization of Latency in Healthcare using Machine Learning
}

\author{
Amit Kishor $^{1 *}$, Chinmay Chakraborty ${ }^{2}$, Wilson Jeberson ${ }^{1}$
${ }^{1}$ Department of Computer Science \& Information Technology, Sam Higginbottom University of Agriculture, Technology and Sciences (SHUATS), Allahabad, U.P. (India)
${ }^{2}$ Assistant Professor, Department of Electronics and Communication Engineering, Birla Institute of \\ Technology, Mesra, Jharkhand (India)
}

Received 28 October 2020 | Accepted 23 December 2020 | Published 21 December 2020

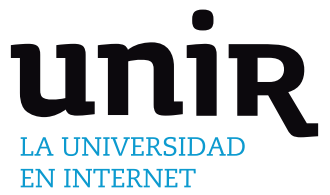

KEYWORDS

Cloud Computing, Data Segregation Scheme, Fog Computing, Latency, Machine Learning, Multimedia Healthcare Data Analytics, Multimedia Transmission, Quality Of Service.

DI: $10.9781 /$ ijimai.2020.12.004

\section{INTRODUCTION}

\begin{abstract}
$\Lambda$ s per the International Data Corporation report, there will be 141.6 billion to 1 trillion IoT devices and that will generate a huge amount of data in zettabytes by 2025 . There is a big demand of wireless communication due to many reasons such as the tremendous increase in the popularity of IoT devices, extensive use of social media, the dissemination of different mobile application, the population growth of the world, and the present lifestyle that is highly dependent on the latest technology in every aspect. A huge number of multimedia data is generated by IoT devices used in healthcare it is very important to process multimedia data in the healthcare sector, Cloud servers are mostly used world-wide to handle the immense data generated by these IoT devices. The extraction of information's about patient health from supplied analyzed multimedia data is plays a very important and crucial role. Analysis of data, storage of data, pre-processing of data is done by cloud servers. Mainly the cloud computing is the probably viable solution for establishing communication between IoT and healthcare [1]. The healthcare data generated by IoT devices is analyzed, filtered, pre-processed and aggregated only on the cloud. Cloud computing has its limitations. As the data transmission rate increases, due to the receiving of these excessive volumes of data, the response time is increasing in the cloud environment. A higher service delay has occurs to end-users. A high volume of data transmission
\end{abstract}

${ }^{*}$ Corresponding author.

E-mail address: amit_kishor@rediffmail.com over the network increases the probability of occurrence of an error and the delay. The loss of data packets and transmission latency is directly related to the quantity of data transmitted through IoT devices to the cloud. Due to this reason, it causes a low quality of service (QoS) produced to the end-user. Cloud computation and data storage are generally not desired in most of the time-sensitive applications of the IoT. Extreme time-bounded problems must be completed nearer to the IoT devices. As the healthcare infrastructures' main requirements are minimization in latency and reduction in network bandwidth, for this it requires data in real-time for a time-critical scenario [2]. The connection is established between end devices and the cloud through routers and gateways. Thus, a huge wide variety of routers are positioned among the cloud and the healthcare IoT's. Due to routers, computational delay increases. As the distance is larger, the large numbers of routers are connected between cloud and IoT devices, because of that a long route is travelled by data from source to destination and it consumes high bandwidth.

For the utilization of the complete advantages of the IoT with fog, it is essential to make available enough networking and infrastructure to produce minimum latency and rapid responding time for IoT applications. Fog computing is introduced as a prime catalyser for the execution and processing of the data generated by IoT devices. It is more effective to shift the applications, execution, and processing capabilities nearer to IoT devices that generated the data. The fog computing concept is properly well suited to resolve these issues.

Fog computing is an emerging concept that uses the processing and execution capabilities closer to the end-user to achieve an improved quality of service as previously used in the cloud paradigm [3]- 
[4]. The fog computing layer is placed in between IoT and cloud; it brings low latency and low network usage. Fog computing provides storage, pre-processing, execution, networking, and computational services to their end-users at the edge of the network and closer to end-users. Despite the number of advantages of fog computing, the available research-work is still immature in this domain, and numbers of researchers are still working on the challenges of fog-computing and basic architecture [3]-[6]. The main challenges of fog devices are privacy, security, and consumption of energy. Fog computing is used to overcome the limitations of cloud computing. Fog Computing is used for such applications that require minimum latency and it works on geo-distribution, which is fast and transferable, and has a broad level distribution control system. It enables distributed and computation with low latency at the edge of the network to provides support to IoT applications. Sufficient amounts of available data can be stored, computed, and processed over the fog-networks and that can be controlled by end-users [7]. An open research aim is to improve the quality of services of fog computing by introducing a fog layer between cloud and IoT devices.

The motivation came from a study about how to generate minimum response time with a better quality of service for time-sensitive healthcare IoT based applications. The cloud alone is not able to satisfy the aforementioned requirements due to their limitations. The patient's physical status varies with time and needs rapid response as an action to monitor remote patients. This is possible when there is a very good network available. Otherwise, it will take more time to respond. In fact, due to unpredictable networks, there is high latency, the health data of patients are not considered as real-time data. This shows that the data become unreliable, worthless, and insufficient. The delay may increase for these IoT time-sensitive data from milliseconds (ms) to seconds and then reaches to minutes [8]-[9], When the size of health data increases, therefore the situation become worsening in handling real-time operation [10]-[11]. The QoS requirements for medical health data [12]-[13] are shown in Table I and the QoS service requirements are shown in Table II for e-healthcare services.

TABLE I. QoS Requirement Parameter for Healthcare and Medical Data Transmission

\begin{tabular}{|c|c|c|c|}
\hline Services for Healthcare & $\begin{array}{c}\text { Data Rate } \\
\text { (kbps) }\end{array}$ & Maximum Delay & $\begin{array}{c}\text { Loss of } \\
\text { Packet (\%) }\end{array}$ \\
\hline Audio & $4.0-25.0$ & $150.0-400.0 \mathrm{~ms}$ & 3.0 \\
\hline Video & $32.0-384.0$ & $150.0-400.0 \mathrm{~ms}$ & 1.0 \\
\hline Electro-cardio-gram (ECG) & $1.0-20.0$ & Approx. 1 second & 0.0 \\
\hline File -Transfer (FTP) & - & - & 0.0 \\
\hline
\end{tabular}

The main contributions of the research work-study are as follows:

1. An analytical model based on fog computing is proposed to transfer healthcare sensor data to end-users in real-time.

2. A random forest algorithm is implemented which reduces and avoids the "over-fitting" issues.

3. The proposed research scheme minimizes the total latency between healthcare sensors and cloud servers. A performance comparison is conducted for the proposed analytical model with existing models on different parameters.

4. To improve the quality of service for e-healthcare.

The remaining part of the paper is organized as follows; Section II describes related work. Section III, introduces a proposed system model for IoT-Fog-Cloud applications. Section IV of the paper is about the work done for the proposed system model. The analysis based on simulation results are provided in Section V. Section VI, comprises the conclusion and provides the future scope of the paper.
TABLE II. QoS Requirement Parameter Services for E-Healthcare

\begin{tabular}{|c|c|c|c|}
\hline $\begin{array}{c}\text { Types of } \\
\text { e-healthcare } \\
\text { services }\end{array}$ & $\begin{array}{c}\text { e-healthcare system } \\
\text { examples }\end{array}$ & $\begin{array}{c}\text { Media type } \\
\text { used }\end{array}$ & $\begin{array}{c}\text { Maximum } \\
\text { delay }\end{array}$ \\
\hline $\begin{array}{c}\text { Audio-based } \\
\text { communication } \\
\text { in real-time }\end{array}$ & $\begin{array}{c}\text { Conferencing (audio) } \\
\text { among patients/end-users } \\
\text { or end-user/doctors }\end{array}$ & Audio & $\begin{array}{c}<150.0 \text { ms one } \\
\text { way end to end }\end{array}$ \\
\hline $\begin{array}{c}\text { Video-based } \\
\text { communication } \\
\text { in real-time }\end{array}$ & $\begin{array}{c}\text { Conferencing (video) } \\
\text { among patients/end-users } \\
\text { or end-user/doctors }\end{array}$ & Video & $\begin{array}{c}<250.0 \text { ms one } \\
\text { way end to end }\end{array}$ \\
\hline $\begin{array}{c}\text { Robotic } \\
\text { services in } \\
\text { real-time }\end{array}$ & $\begin{array}{c}\text { Remote based tele- } \\
\text { surgery }\end{array}$ & $\begin{array}{c}\text { Control of data, } \\
\text { audio, video } \\
\text { by robotics }\end{array}$ & $\begin{array}{c}<300.0 \\
\text { milliseconds } \\
\text { round trip time }\end{array}$ \\
\hline $\begin{array}{c}\text { Monitoring in } \\
\text { real-time }\end{array}$ & $\begin{array}{c}\text { Patient's essential sign } \\
\text { transmission and video } \\
\text { steaming in an urgent } \\
\text { scenario }\end{array}$ & $\begin{array}{c}\text { Sensors } \\
\text { (to collect } \\
\text { biomedical } \\
\text { data) }\end{array}$ & $\begin{array}{c}<300.0 \\
\text { milliseconds for } \\
\text { real-time ECG }\end{array}$ \\
\hline $\begin{array}{c}\text { Real-time } \\
\text { diagnosis }\end{array}$ & $\begin{array}{c}\text { Transfer the medical } \\
\text { images to remote areas in } \\
\text { an urgent scenario }\end{array}$ & $\begin{array}{c}\text { Images, text, } \\
\text { data }\end{array}$ & - \\
\hline $\begin{array}{c}\text { Real-time } \\
\text { messaging }\end{array}$ & $\begin{array}{c}\text { Alarm based indication } \\
\text { for urgency }\end{array}$ & $\begin{array}{c}\text { Text, data, } \\
\text { small images }\end{array}$ & No \\
\hline
\end{tabular}

\section{RELATED WORK}

Silva et al. [14] used fog computing technology to manage patient records. Fog computing is used to overcome the problem of cloud computing for data management with challenges such as availability, performance, and secrecy. Alarm et al. [15] proposed a method to store the health data on the cloud. The data is generated by IoT-devices. They presented a system for data management in the cloud, based on the management of IoT. The collection of data is done in real-time and an alert system is there with a prior defined rule for notification. Nishtala et al. [16] used a combination of heuristic and reinforcement learning technology called Hipster used to control the latency-critical workloads in the cloud. Hipster aims to improve the efficiency of used resources concerning the quality of service. Latency for large computations is not discussed by the author for the cloud environment. Gia et al. [17] proposed research for continuous monitoring of timesensitive health patient's through fog computing and concern cost is low. They provided automatic notification and analysis. The sensornode (energy efficient) system is developed with a layer of fog. Medical practitioners access the data collected through sensors. Naas et al. [18] raised the major problem for IoT applications in time-sensitive cases which is resolved by the author with a technique proposed named iFogStor in fog environment. The author proposed a schema called GAP (Generalized Assignment Problem) for the placement of the data in fog computing. For the solution of GAP two methods are used, first is an accurate solution and the other one is the heuristics method. Rahmani et al. [19] discussed the different services such as real-time processing of local data, data-mining (embedded), and some higherlevel services. They presented a prototype called UT-GATE for smart e-health gateway and through which they discussed the features. They have shown the enhancement in performance of overall systems. Wu et al. [20] proposed a schema as security services in fog computing in information-concentric social networks. The main contribution is the introduction of fog computing concepts with required parameters end-to-end communication, low latency, and computing resources at the network edge and also improving the security services by content-aware and matching. Although the network delays, as well as computation delay, are not being discussed by the author. Brogi et al. [21] presented a model for the deployment of QoS-aware in IoT used applications by the use of fog computing technology. The model 
is used to produce the latency and bandwidth of accessible resources but the author missed discussing network and computation latency.

Shahzad et al. [22] proposed a method to monitor the medical condition in real-time compare to the private cloud. A system is designed and known as BTS (bounded telemonitoring system) for monitoring of patients in real-time. The information for patients is captured in the boundary of the private cloud. They try to provide medical data of patient's with security. Kao et al. [23] introduced the time-critical data analysis in mobile computing for latency minimization the author presented a novel technique with the name of Hermes. The optimization technique based on NP-hard is used for the task data. Li et al. [24] introduced the SPSRP's (service popularitybased smart-resources partitioning) architecture for implementation in IoT and fog computing and also created a mathematical model for the popularity of service and cost of computation on Fog Nodes (FNs). The authors reduced fault tolerance, response-time, and delay time. The calculation for the cost of computing on FNs at the arrival of services from IoT by applying Zipf's law is provided.

Dinh et al. [25] used a service-oriented schema related to costeffectiveness for providing the service of the IoT-Fog-Cloud network. The authors also used to measure VNF (Virtual Network Function) with development in the capabilities to enhance the availability of SFC (service function chaining) with the proposed metric. Mahmud et al. [26] discussed the problem that occurred in the use of healthcare due to the large volume of transmission of data and high latency. As a solution to these issues, the author presented an IoT-healthcare structure based on fog and explored the cloud-fog service over the traditional cloud. An improvement result is shown for network-traffic, power usage, and the cost. Ahsan et al. [27] highlighted the security, protection, and integrity of the data is a major concern in cloud computing. The author proposed a fog-centric scheme for the storage of data in the cloud. Data security issues had been discussed. Xorcombination is implemented to provide the protection and security of data in the cloud. The proposed method is used to prevent the attack of unauthorized access and malicious users. Hash technique was used in a new form to detect the data alteration with a high occurrence of probability. They also prevented a cyber attack. Rafique et al. [28] used a technique with modification and combination of the PSO (Particle Swarm Optimization) and CSO (Cat Swarm Optimization) to reduce the response time. With the combination of the above two algorithms, they produce NBIHA (Novel bio-inspired hybrid-algorithm) used to overcome the response-time in IoT-Fog-Cloud applications. $\mathrm{Li}$ et al. [29] introduced the factors of network delays and designed a framework based on IoT-Fog for estimation of latency. They can predict the delay occurred in the cloud-fog inter-node and proposed a GNP (Global Networking Position) a landmark-based algorithm to predict the latency with good accuracy. Thota et al. [30] presented sensor architecture by using a fog computing platform. Sensors were used to collect patient data and after that sensor send data to edge devices with security. They provided authentication and security of medical data, and unauthorized access was prevented by using asynchronous communication.

Tahani [31], used the scheduling algorithm MAX-MIN on medical data, and then the author used a new method for distribution of task to reduce the waiting time in queue, called TCVC (Task Classification and Virtual Machine Categorization). Raafat et al. [32], presented a model for resource allocation in fog and cloud environments when the data is generated by edge devices. They calculated the overall latency of the model in a fog environment using a genetic algorithm. Pan et al. [33], presented and discussed the current technologies summary report and the compatibility among the cloudlet, home cloud, nebula, fog computing, MEC (mobile-edge-computing). They discussed the different issues related to the aforementioned technologies. But no practical issue is discussed. Chakraborty et al. [36]-[37] measure QoS over heterogeneous networks. Nilashi et al.[38] presented a heart disease prediction model by using fuzzy-SVM. They improve accuracy and computation time. Tarik et al. [39] presented a model for diabetic patients. They analyzed the fasting blood sugar as attributes

TABLE III. EXIsting Literature SURVEY

\begin{tabular}{|c|c|c|c|}
\hline Authors & Proposed Techniques & Advantages & Limitations \\
\hline $\begin{array}{l}\text { Alam et al. } \\
\text { [15] }\end{array}$ & A real-time data collection in fog computing & $\begin{array}{l}\text { Data collected in real-time. Transmission delay and } \\
\text { computation delay is calculated }\end{array}$ & $\begin{array}{l}\text { There is no calculation for network } \\
\text { delay }\end{array}$ \\
\hline $\begin{array}{l}\text { Nishtala et } \\
\text { al. [16] }\end{array}$ & Hipster : to control time-sensitive issues & $\begin{array}{l}\text { Improved efficiency by using network and } \\
\text { computation delay }\end{array}$ & $\begin{array}{l}\text { Latency for large data is not } \\
\text { discussed and also no method is } \\
\text { designed for transmission delay }\end{array}$ \\
\hline $\begin{array}{l}\text { Naas et al. } \\
\quad[18]\end{array}$ & $\begin{array}{l}\text { iFogStor : GAP for fog computing and heuristic } \\
\text { approach }\end{array}$ & $\begin{array}{l}\text { System efficiency is improved. Also they resolve the } \\
\text { issue occurred with time-sensitive data. }\end{array}$ & $\begin{array}{c}\text { The transmission delay is not } \\
\text { calculated }\end{array}$ \\
\hline $\begin{array}{l}\text { Brogi et al. } \\
\quad[21]\end{array}$ & $\begin{array}{c}\text { QoS-aware model deployment in IoT by fog } \\
\text { computing }\end{array}$ & Deployed a QoS-aware model in IoT & $\begin{array}{l}\text { No explanation for network and } \\
\text { computation delay }\end{array}$ \\
\hline $\begin{array}{l}\text { Shahzad et } \\
\text { al. [23] }\end{array}$ & Hermes: NP-hard technique & Task data is optimized by using NP-hard & $\begin{array}{l}\text { There is no explanation for network } \\
\text { and computation delay }\end{array}$ \\
\hline $\begin{array}{l}\text { Li et al. } \\
{[24]}\end{array}$ & SPSRP for fog nodes (FNs) and IoT-device & $\begin{array}{c}\text { Minimizes the response and delay time in the fog } \\
\text { environment }\end{array}$ & $\begin{array}{l}\text { Computation and network delay is } \\
\text { not discussed }\end{array}$ \\
\hline $\begin{array}{l}\text { Mahmud } \\
\text { et al. [26] }\end{array}$ & IoT-healthcare structure for cloud-fog & $\begin{array}{l}\text { Discusses the issue of high volume data. Improved the } \\
\text { performance of network traffic and power }\end{array}$ & $\begin{array}{l}\text { Network delay is not being } \\
\text { discussed }\end{array}$ \\
\hline $\begin{array}{l}\text { Ahsan et } \\
\text { al. [27] }\end{array}$ & A fog-centric schema for data storage & Discusses the storage and security of data in the cloud & $\begin{array}{l}\text { No discussion about transmission } \\
\text { and network delay }\end{array}$ \\
\hline $\begin{array}{l}\text { Rafique et } \\
\text { al. [28] }\end{array}$ & PSO and CSO techniques & $\begin{array}{c}\text { Reduces the response time in the IoT-Fog-Cloud } \\
\text { environment }\end{array}$ & $\begin{array}{l}\text { There is no discussion about } \\
\text { network and computation delay }\end{array}$ \\
\hline $\begin{array}{l}\text { Proposed } \\
\text { scheme }\end{array}$ & IMDS & $\begin{array}{l}\text { Reduce the overall latency by using } \\
\text { transmission, network, and computation delay }\end{array}$ & - \\
\hline
\end{tabular}


for predicting of the diabetics. Mahmud et al. [40] highlighted the recent techniques to capture the different types of patient data in the research. They also captured the video data of the patients. Tarik et al. [41] presented a method for healthcare analysis of patients through meta-heuristic algorithms. This method is very useful for doctors and patients when the patients are suffering from different diseases. Jerry et al., [42] presented a model named BILU-NEMH for extraction and classification of data. They used hypergraph and deep learning concepts to enhance the performance of the designed model. Jerry et al. [43], highlighted the problem faced by sequence labeling and they proposed a model for enhancing the sequence labeling with latent variable conditional random fields. This model is very useful in the stage of the pre-processing of data. Machine understanding becomes strengthens through this model. Ahmed et al. [44], presented a machine learning-based classifier, and the method is mapped with OpenCL. The classification can be accelerated by the use of the proposed method for heterogeneous networks. They also highlighted the solution method for the data imbalance problem. Table III shows the survey on existing literature and Table IV shows the comparative analysis.

TABLE IV. The Comparative Analysis

\begin{tabular}{|l|c|c|c|}
\hline Authors/Year & $\begin{array}{c}\text { Transmission } \\
\text { Delay }\left(\mathbf{T}_{\mathrm{D}}\right)\end{array}$ & $\begin{array}{c}\text { Network } \\
\text { Delay }\left(\mathbf{N}_{\mathrm{D}}\right)\end{array}$ & $\begin{array}{c}\text { Computation } \\
\text { Delay }\left(\mathbf{C}_{\mathrm{D}}\right)\end{array}$ \\
\hline Alam et al. [15], 2016 & Yes & No & Yes \\
\hline Nishtala et al. [16], 2017 & No & Yes & Yes \\
\hline Naas et al. [18], 2017 & No & Yes & Yes \\
\hline Wu et al. [20], 2017 & Yes & No & No \\
\hline Brogi et al. [21], 2017 & Yes & No & No \\
\hline Shahzad et al. [23], 2017 & Yes & No & No \\
\hline Li et al. [24], 2018 & Yes & No & No \\
\hline Dinh et al. [25], 2018 & Yes & No & No \\
\hline Mahmud et al. [26], 2018 & Yes & Yes & No \\
\hline Ahsan et al. [27], 2019 & No & No & Yes \\
\hline Rafique et al. [28], 2019 & Yes & No & No \\
\hline $\begin{array}{l}\text { Proposed IMDS } \\
\text { Algorithm }\end{array}$ & Yes & Yes & Yes \\
\hline
\end{tabular}

After analyzing the available research and studying the comparative comprehensive analysis of the reduction in total latency (transmission delay, network delay, and computation delay) among IoT-Fog-Cloud networks, we found that there is a research gap and the available techniques for reducing the latency in healthcare used by the researcher are incomplete. Hence, a novel technique must be required to fill this research gap.

To achieve the imperative execution, the issue of minimization of latency in healthcare cloud and IoT was developed and for the aforementioned aim the system model is presented, the main aim is for the formation of the fog network, to effectively allocate, and distribute the task data. To create the network of fog and unload its task data, a fog node (FN) should search neighboring or adjacent FNs. The neighboring FNs in the system will dynamically appear and disappear. It is well known that, In the healthcare system for monitoring highrisk patients, regular monitoring of patients is required. To maintain the regular monitoring system by the human being is very difficult, tedious and it seems to be an unpractical approach. As a result, carelessness towards the high-risk patient occurs. To avoid such situations, the aim is to evaluate the patient health data to track the
TABle V. Description of Data Used in the Proposed Model

\begin{tabular}{|c|c|c|}
\hline Sr. No. & Variable Name & Description \\
\hline 1 & Age & Patient's age ( in years) \\
\hline 2 & Sex & Male/Female as $1 / 0$ \\
\hline 3 & $\mathrm{CP}$ & $\begin{array}{l}\text { Chest Pain type (result } 1 \text { : Angina, result } 2: \text { A } \\
\text { typical way of Angina, result } 3 \text { : Not-angina, } \\
\text { result } 4 \text { : Angina symptom nil) }\end{array}$ \\
\hline 4 & Trestbps & Blood Pressure values in resting in $\mathrm{mm} \mathrm{Hg}$ \\
\hline 5 & Chol & Cholesterol results in $\mathrm{mg} / \mathrm{dl}$ \\
\hline 6 & FBS & $\begin{array}{l}\text { Blood Sugar results in fasting }>120 \mathrm{mg} / \mathrm{dl} \text { ( } 1 \text { as } \\
\text { true; } 0 \text { as false) }\end{array}$ \\
\hline 7 & Restecg & $\begin{array}{l}\text { ECG resting results (result } 0 \text { for normal; result } 1 \\
\text { and } 2 \text { for abnormal) }\end{array}$ \\
\hline 8 & Thalach & Heart Rate ( maximum) as recorded \\
\hline 9 & Exang & Prompted Angina exercise( 1 as yes; 0 as no) \\
\hline 10 & Oldpeak & $\begin{array}{l}\text { ST Depression prompted by Exercise as } \\
\text { compare to rest }\end{array}$ \\
\hline 11 & Slope & $\begin{array}{l}\text { The slope respect to peak of exercise (result 1,2, } \\
\text { and } 3 \text { for up sloping, flat, and downsloping) }\end{array}$ \\
\hline 12 & $\mathrm{CA}$ & Major vessels number (total) \\
\hline 13 & Thalrest & Values (at rest) of heart rate \\
\hline 14 & NUM & $\begin{array}{l}\text { Status of heart disease } \\
\text { (result } 0=\text { no heart disease; } \\
\text { result greater than } 0=\text { heart disease) }\end{array}$ \\
\hline
\end{tabular}

probability of any high risk, the system required an analysis of a large volume of healthcare data set and attributes. Random forest is applied for the detection, segregation, and analysis of data. Random forest is selected to avoid the over-fitting problem. The predicted data is sent to the end-user within minimum time. Here, to find the availability of adjacent FN for computation is very difficult. In addition to it, the total numbers of adjacent or neighboring FNs with their locations are unidentified and extremely unpredictable, so it is very challenging to manage the fog network creation and task data distribution process. So under such an unpredictable condition, also considering neighboring FNs is accountable for the appearance of new FNs, which also produces a higher data rate and increasing computational capabilities.

\section{Proposed Model}

The Fog computing environment based IoT- healthcare system model shown in Fig. 1. The proposed model collects the patient data as per table $\mathrm{V}$. The data is transmitted from IoT or sensor devices and then data is classified into three categories such as low sensitive risk, normal, and high sensitive risk by applying random forest machine learning algorithm. Healthcare sensor data offload their task data to fog servers. After processing healthcare data the time-sensitive data are sent to the end-user in minimum time. FNs are used to distribute and allocate the task data packets in different available nodes and end-user. A principal FN manager is used and that maintains the topological details of task data packet distribution and allocation. Network topology is used to connect the nodes and every FNs are then linked with the principal FN. Here the study shows a continual 
task data packet allocation system using fog computing environment in machine learning. FNs transfer the task data packet to other FNs in the network to minimize the latency and reduce the network traffic. Here, the processing unit comprises the task data packets in a transmission queue, and then the task data is sent for computation in the computation queue, and these impacts the response time. The entire FN collects details, composes a decision, provides task data information and maintains the queue position. A network table is created by the principal FN and it considers all information that was distributed by the other nodes. The principal FN sends a request to other FNs to determine their availability to process the required data. After getting the availability, the task data will be sent to the nearest FN, where the allotment is performed based on requisite data and time. The work aims to reduce or minimize latency and traffic of network with the selection of time-sensitive data. The process of interacting is that IoT sends data to FN and received data is served by the same FN if data is small otherwise FN serves it partially and sends the remaining data to neighboring FNs to serve. The neighboring FNs compute the data if they are not currently processing any data otherwise data have to wait in the waiting queue for processing. After processing of data from neighboring nodes, these send back the data to the original FN (which transfers the task data first) then the FN sends it to the enduser or cloud. Therefore the selection of the best neighboring nodes is very important for task processing otherwise it increases the waiting time in the computation queue.

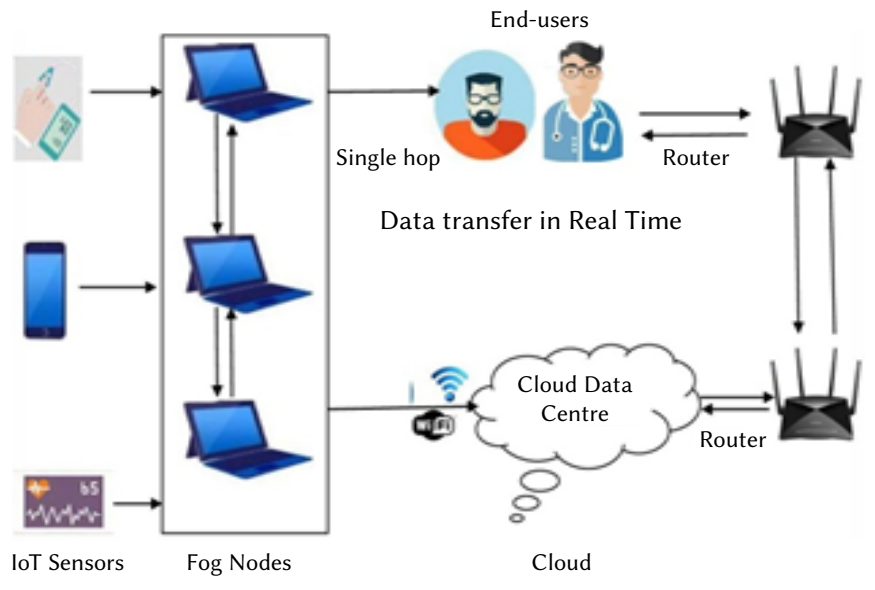

Fig. 1. IoT- HealthCare System Model.

The principal node will maintain an availability table in which all the detail of FNs will be available and maintain all the current updates of the status of FNs. The availability of nodes is shown in table VI, containing the approximate waiting time and the processing speeds of FNs. If any FN became free early then it updates the approximately waiting time in the availability nodes table. The principal node will regularly update the approximately waiting time. The selection of best neighboring FN can be performed by selecting the highest processing speed with a minimum of approximately waiting time.

TABLE VI. Availability of Nodes

\begin{tabular}{|c|c|c|}
\hline $\begin{array}{c}\text { Node } \\
\text { Description }\end{array}$ & $\begin{array}{c}\text { Approx Weighting Time } \\
\text { (microseconds) }\end{array}$ & $\begin{array}{c}\text { The processing speed } \\
\text { of FNs (in G.Hz) }\end{array}$ \\
\hline 132.115 .76 .84 & 245 & 2.201 \\
\hline 132.115 .76 .86 & 237 & 2.202 \\
\hline$\ldots \ldots \ldots$ & $\ldots \ldots$ & $\ldots \ldots$ \\
\hline$\ldots \ldots \ldots$ & $\ldots \ldots$ & $\ldots \ldots$ \\
\hline
\end{tabular}

The random forest machine learning algorithm [34]-[35] was used to achieve the aim of the minimization of latency. This aim can be achieved by reducing the used delay as transmission, network, and computation. The proposed model used IoT-Fog-Cloud application's dynamic behavior. A decision-oriented process has been performed using a random forest machine-learning algorithm to overcome the issue of task data demand at a distinct time interval among distinct users and the processing capabilities of FNs. In real-time, the random forest algorithms are used to monitor and care the health data. The main purpose is to minimize the delay that occurs in health monitoring. The FNs identify and select best neighboring FN for computation and process. The quality of service is also a major concern of the entire system. The FNs were used to select the task data communicated by the IoT application in the proposed research. Thereafter, it starts the processing of health task data, and the remaining part of data is transferred to the best neighboring FN and then these processed data is sent to either end-user or cloud in real-time. All the executions are required to be processed in minimum time.

\section{MATERIALS AND METHODS}

Healthcare heart disease data are taken from UC Irvine's machine learning repository [46]. In the simulation heart disease data set encompasses 303 instances and 14 attributes. Although, the UCI repository encompasses 76 attributes in the actual heart disease dataset. In total 14 attributes have been taken for simulation of the proposed algorithm. The testing of the algorithm is performed on these attributes. The attributes are categorized into qualitative and quantitative attributes as shown in the Table V, which shows the data description used in the proposed model. The selection of high-risk data is based on qualitative attributes.

To achieve the objective of the research we applied a k-fold random forest machine learning algorithm. The reason to apply random forest is having better contributions among other classifiers such as SVM (support vector machine), BN (Bayes Network), MP (Multilayer perception), etc. [33]. Feature selection becomes easier in a random forest based algorithm. Estimation of missing values is completed effectively. It avoids over-fitting problems despite that it is a collection of decision trees. Many of research work said that random forest has a quality for prediction of accuracy is excellent for both normal and abnormal data. In a random forest method, the optimization of features is governed by bootstrapped data and this can be performed by k-fold cross-validation $(\mathrm{k}=10)$. To avoid overfitting the other scheme such as early stopping and ensembling can also be used. Fig. 2 represents the flow chart of the intelligent multimedia data segregation (IMDS) scheme. The distance travel and the number of hops covered from the sensor to the cloud server is minimum for the high-risk data because it is processed near to sensor devices known as fog computing. By the use of this process, there is a reduction in transmission time due to the total latency time reduces.

In the proposed IMDS algorithm based on k-fold random forest machine learning techniques, the model collects the data at the initial level. Data is pre-processed after collection. Then data is divided into $\mathrm{k}$-fold. Herein k-fold cross-validation is applied. The cross-validation process is evaluating the model by dividing the original sample into small k-chunks. The partition process of the original data in k-chunks used a random approach but the size is always equal. In k-fold, k-1 chunks are used for training the model, and the remaining single chunk is used to test the model. The Gini index is calculated for accurate measurement. Training and testing of data are completed with a ratio of 70 and 30 . We can also train our proposed model by using meta-heuristic optimization techniques [45]. 


\author{
Proposed IMDS Algorithm: \\ 1. from random import randrange \\ 2. from csv import reader \\ 3. from math import sqrt \\ 4. defload_csv(filename): \\ 5. def str_column_to_float(dataset, column): \\ 6. defstr_column_to_int(dataset, column): \\ 7. def cross_validation_split(dataset, $n \_$folds): \\ 8. dataset_split $=$ list () \\ 9. dataset_copy $=$ list (dataset) \\ 10. fold_size $=$ int $\left(\right.$ len $($ dataset $) / n \_$folds $)$ \\ 11. def test_split(index, value, dataset): \\ 12. binwidth $=\operatorname{int}((\max (d f[$ "survival_score"] $)$ - \\ $\min (d f[$ "survival_score"]))/3)bins=range(min (df["survival_ \\ score"]), max(df["survival_score"],binwidth) \\ group_name $=$ ['normal',low_risk','high_risk'] \\ 13. def gini_index(groups, classes): \\ 14. gini $+=(1.0-$ score $){ }^{*}($ size $/ n$ _instances $)$ \\ 15. return gini \\ 16.defbuild_tree(train, max_depth, min_size, $\left.n \_f e a t u r e s\right)$ : \\ 17. root = get_split(train, $n \_$features) \\ 18. split(root, max_depth, min_size, n_features, 1) \\ 19. return root \\ 20. def predict(node, row): \\ 21. if row [node ['index']] < node['value']:

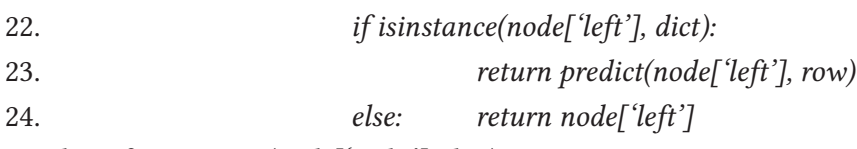

25. else: if is instance(node['right'], dict):

26. return predict(node['right'], row)

27. else: return node['right']

28. def random_forest(train, test, max_depth, min_size, sample_size, $n \_$trees, $n \_$features):

29. trees $=$ list ()

30. for $i$ in range(n_trees):

31.

sample $=$ subsample(train, sample_size $)$

32. min_size, $n$ features)

33. trees.append(tree)

34. predictions $=$ [bagging_predict(trees, row) for row in test $]$

35. return(predictions)

36. filename $=$ 'sonar.all-data.csv'

37. dataset $=$ load_csv(filename)

38. for $i$ in range( 0 , len(dataset [0])-1):

$39 . \quad$ str_column_to_float(dataset, $i)$ str_column_to_int(dataset, len(dataset [0])-1)

$n \_$folds $=10$, max_depth $=10$, min_size $=1$, sample_size $=1.0$ $n \_$features $=\operatorname{int}(\operatorname{sqrt}($ len $($ dataset $[0])-1))$

40. for n_trees in $[1,10,10]$ :
41. scores $=$ evaluate algorithm(dataset, random_forest, $\quad n$ folds, max_depth, min_size, sample_size, $n \_t r e e s, n$ features)

42. print('Mean Accuracy: \%.3f\%\%'\% (sum(scores)/float(len(scores))))

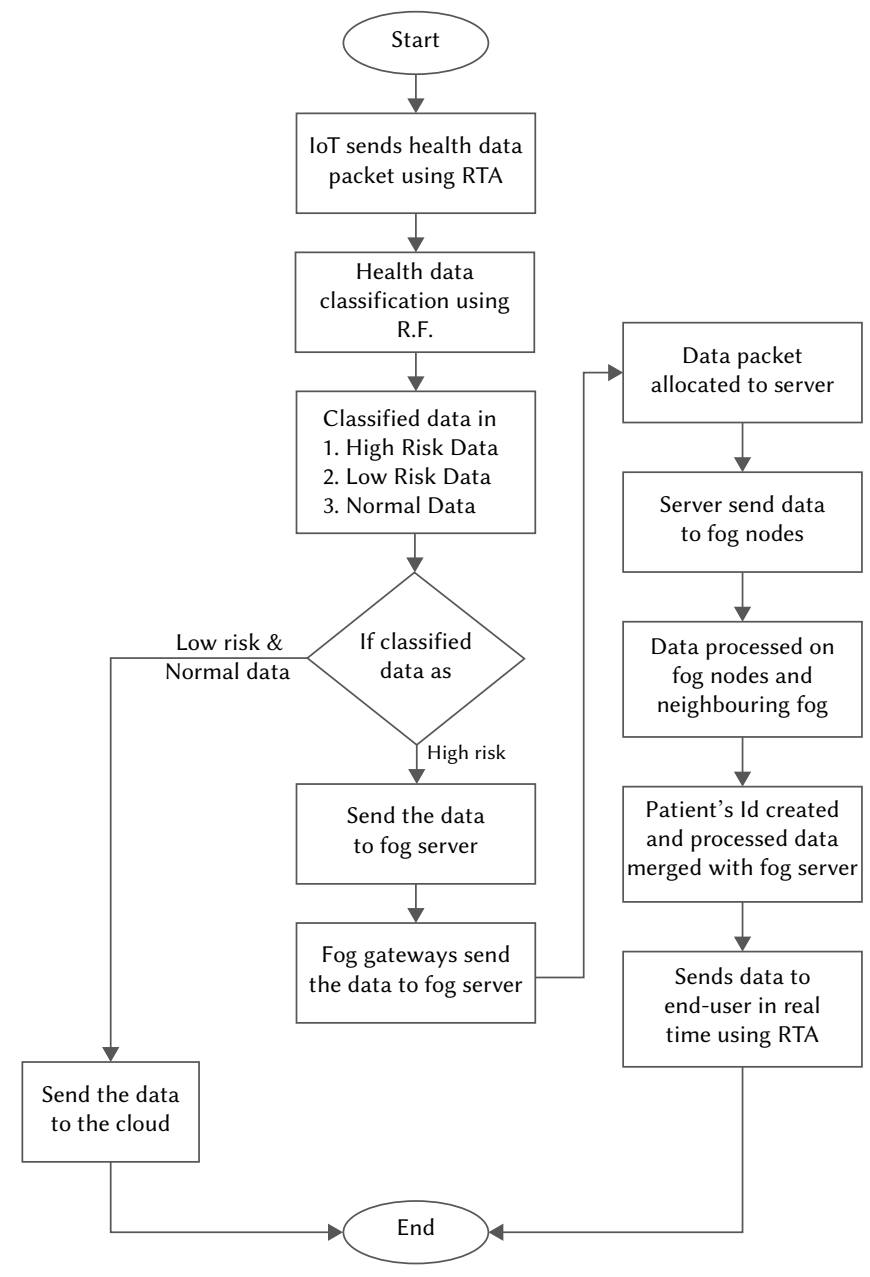

Fig. 2. Flow chart for IMDS scheme.

\section{A. A mathematical Framework For Latency Calculation}

Here we assume that there are different kinds of sensors used to forwarding their health data to an FN. In this research, for implementation of fog computing, we are concern with finding the low latency in health informatics. Considering a fog network, containing a cloud layer, a fog layer, and a sensor layer, here the data will be transferred regularly between all the tiers. The sensor layer is containing smart in nature and small in size IoT devices and they don't have enough capability of computations. The fog networks are placed closer to IoT devices to process the patient's data. It is considered that different kinds of sensor devices send their data to a FN (i) and the data size will be XP packets/second. FN (i) performs the task of controlling, storing, analyzing, and processing the health data received from sensors. Here the FNs (i) cooperate with other adjacent or neighboring nodes. After receiving XP packets of the task at FN (i) from the end-user node (e) then the FN distributes the task to adjacent or neighboring FNs ( $\mathrm{j}$ ) for computation. After computation the task is returned to the main FN. Here, the transmission delay for the request of FN and response time from neighboring FN is calculated as the transmission delay.

Transmission delay $\left(\mathrm{T}_{\mathrm{D}}\right)$ : Transmission delay $\left(\mathrm{T}_{\mathrm{D}}\right)$ is the round trip time (RTT) in relaying of data fragment between end-users nodes (wearable sensors) to FNs can be calculated by $\mathrm{M} / \mathrm{D} / 1$ system as follows

$$
\mathrm{T}_{\mathrm{D}}=\mathrm{FN}_{\mathrm{RT}}+\mathrm{FN}_{\mathrm{RPT}}
$$

Transmission delay between end-user node (e) to FN (i) is as follows $\mathrm{FN}$ request $\left(\mathrm{FN}_{\mathrm{RT}}\right)$ from e to $\mathrm{i}$ is, 


$$
\mathrm{T}_{e i}=\frac{\lambda_{\mathrm{ei}}}{2 \mu_{e i}\left(\mu_{e i}-\lambda_{e i}\right)}+\frac{1}{\mu_{e i}}
$$

$\mathrm{FN}$ response time $\left(\mathrm{FN}_{\mathrm{RPT}}\right)$ from i to e is,

$$
\mathrm{T}_{\mathrm{ie}}=\frac{\lambda_{\mathrm{ie}}}{2 \mu_{i e}\left(\mu_{i e}-\lambda_{i e}\right)}+\frac{1}{\mu_{i e}}
$$

Where

$$
\begin{aligned}
& \mu_{\mathrm{ei}}=\mathrm{B}_{\mathrm{e}} \log _{2}\left(1+\frac{g_{e i} P_{t x, e}}{B_{e} N_{o}^{e}}\right) \\
& \text { and } g_{e i}=\gamma_{1} d_{e i}^{-\gamma_{2}} \\
& \mu_{\mathrm{ie}}=\mathrm{B}_{\mathrm{i}} \log _{2}\left(1+\frac{g_{i e} P_{t x, i}}{B_{i} N_{o}^{i}}\right) \\
& \text { and } g_{i e}=\gamma_{3} d_{i e}^{-\gamma_{4}}
\end{aligned}
$$

Hence the transmission delay is as

$$
\begin{aligned}
& \mathrm{T}_{\mathrm{D} 1}=\mathrm{T}_{\mathrm{ei}}+\mathrm{T}_{\mathrm{ie}} \\
& =\frac{\lambda_{\mathrm{ei}}}{2 \mu_{e i}\left(\mu_{e i}-\lambda_{e i}\right)}+\frac{1}{\mu_{e i}}+\frac{\lambda_{\mathrm{ie}}}{2 \mu_{i e}\left(\mu_{i e}-\lambda_{i e}\right)}+\frac{1}{\mu_{i e}} \\
& =\frac{\lambda_{\mathrm{ei}}}{2 \mu_{e i}\left(\mu_{e i}-\lambda_{e i}\right)}+\frac{\lambda_{\mathrm{ie}}}{2 \mu_{i e}\left(\mu_{i e}-\lambda_{i e}\right)}+\frac{1}{\mu_{e i}}+\frac{1}{\mu_{i e}}
\end{aligned}
$$

$\mathrm{T}_{\mathrm{D}}$ between FN (i) and neighboring node (j) can be expressed as follows

$\mathrm{FN}$ request $\left(\mathrm{FN}_{\mathrm{RT}}\right)$ from $\mathrm{i}$ to $\mathrm{j}$ is,

$$
\mathrm{T}_{\mathrm{ij}}=\frac{\lambda_{\mathrm{ij}}}{2 \mu_{i j}\left(\mu_{i j}-\lambda_{i j}\right)}+\frac{1}{\mu_{i j}}
$$

$\mathrm{FN}$ response time $\left(\mathrm{FN}_{\mathrm{RPT}}\right)$ from $\mathrm{j}$ to $\mathrm{i}$ is,

$$
\begin{aligned}
& \mathrm{T}_{\mathrm{ji}}=\frac{\lambda_{\mathrm{ji}}}{2 \mu_{j i}\left(\mu_{j i}-\lambda_{j i}\right)}+\frac{1}{\mu_{j i}} \\
& \text { Where } \mu_{\mathrm{ij}}=\mathrm{B}_{\mathrm{j}} \log _{2}\left(1+\frac{g_{j i} P_{t x, j}}{B_{j} N_{o}^{j}}\right) \\
& \text { And } g_{i j}=\gamma_{5} d_{i j}^{-\gamma_{6}} \\
& \mu_{\mathrm{ji}}=\mathrm{B}_{\mathrm{i}} \log _{2}\left(1+\frac{g_{i j} P_{t x, i}}{B_{i} N_{o}^{i}}\right) \\
& \text { And } g_{j i}=\gamma_{7} d_{j i}^{-\gamma_{8}}
\end{aligned}
$$

Hence the transmission delay is as

$$
\mathrm{T}_{\mathrm{D} 2}=\frac{\lambda_{\mathrm{ij}}}{2 \mu_{i j}\left(\mu_{i j}-\lambda_{i j}\right)}+\frac{\lambda_{\mathrm{ji}}}{2 \mu_{j i}\left(\mu_{j i}-\lambda_{j i}\right)}+\frac{1}{\mu_{j i}}+\frac{1}{\mu_{i j}}
$$

Transmission delay between end user node (e) and neighboring FN (j) is

$\mathrm{FN}$ request $\left(\mathrm{FN}_{\mathrm{RT}}\right)$ from $\mathrm{i}$ to $\mathrm{j}$ is

$$
\mathrm{T}_{\mathrm{ej}}=\frac{\lambda_{\mathrm{ej}}}{2 \mu_{e j}\left(\mu_{e j}-\lambda_{e j}\right)}+\frac{1}{\mu_{e j}}
$$

$\mathrm{FN}$ response time $\left(\mathrm{FN}_{\mathrm{RPT}}\right)$ from $\mathrm{i}$ to e is,

$$
\begin{aligned}
& \mathrm{T}_{\mathrm{je}}=\frac{\lambda_{\mathrm{je}}}{2 \mu_{j e}\left(\mu_{j e}-\lambda_{j e}\right)}+\frac{1}{\mu_{j e}} \\
& \text { Where, } \mu_{\mathrm{je}}=\mathrm{B}_{\mathrm{j}} \log _{2}\left(1+\frac{g_{j e} P_{t x, j}}{B_{j} N_{o}^{j}}\right) \\
& \text { And } g_{e j}=\gamma_{9} d_{e j}^{-\beta \gamma_{10}} \\
& \mu_{\mathrm{ej}}=\mathrm{B}_{\mathrm{e}} \log _{2}\left(1+\frac{g_{e j} P_{t x, e}}{B_{e} N_{o}^{e}}\right)
\end{aligned}
$$

$$
g_{j e}=\gamma_{11} d_{j e}^{-\gamma_{12}}
$$

The transmission delay between the end node (e) and the neighboring $\mathrm{FN}(\mathrm{j})$ is

$$
\mathrm{T}_{\mathrm{D} 3}=\frac{\lambda_{\mathrm{ej}}}{2 \mu_{e j}\left(\mu_{e j}-\lambda_{e j}\right)}+\frac{\lambda_{\mathrm{je}}}{2 \mu_{j e}\left(\mu_{j e}-\lambda_{j e}\right)}+\frac{1}{\mu_{e j}}+\frac{1}{\mu_{j e}}
$$

Hence the total transmission delay will be

$$
\begin{aligned}
& \mathrm{T}_{\mathrm{D}}=\mathrm{T}_{\mathrm{D} 1}+\mathrm{T}_{\mathrm{D} 2}+\mathrm{T}_{\mathrm{D} 3} \\
& =\frac{\lambda_{\mathrm{ei}}}{2 \mu_{e i}\left(\mu_{e i}-\lambda_{e i}\right)}+\frac{\lambda_{\mathrm{ie}}}{2 \mu_{i e}\left(\mu_{i e}-\lambda_{i e}\right)}+\frac{1}{\mu_{e i}}+\frac{1}{\mu_{i e}}+\frac{\lambda_{\mathrm{ij}}}{2 \mu_{i j}\left(\mu_{i j}-\lambda_{i j}\right)}+ \\
& \frac{\lambda_{\mathrm{ji}}}{2 \mu_{j i}\left(\mu_{j i}-\lambda_{j i}\right)}+\frac{1}{\mu_{j i}}+\frac{1}{\mu_{i j}}+\frac{\lambda_{\mathrm{ej}}}{2 \mu_{e j}\left(\mu_{e j}-\lambda_{e j}\right)}+ \\
& \frac{\lambda_{\mathrm{je}}}{2 \mu_{j e}\left(\mu_{j e}-\lambda_{j e}\right)}+\frac{1}{\mu_{e j}}+\frac{1}{\mu_{j e}} \\
& =\frac{\lambda_{\mathrm{ei}}}{2 \mu_{e i}\left(\mu_{e i}-\lambda_{e i}\right)}+\frac{\lambda_{\mathrm{ie}}}{2 \mu_{i e}\left(\mu_{i e}-\lambda_{i e}\right)}+\frac{\lambda_{\mathrm{ij}}}{2 \mu_{i j}\left(\mu_{i j}-\lambda_{i j}\right)}+ \\
& \frac{\lambda_{\mathrm{ji}}}{2 \mu_{j i}\left(\mu_{j i}-\lambda_{j i}\right)}+\frac{\lambda_{\mathrm{ej}}}{2 \mu_{e j}\left(\mu_{e j}-\lambda_{e j}\right)}+\frac{\lambda_{\mathrm{je}}}{2 \mu_{j e}\left(\mu_{j e}-\lambda_{j e}\right)}+\frac{1}{\mu_{e i}}+\frac{1}{\mu_{i e}} \\
& +\frac{1}{\mu_{j i}}+\frac{1}{\mu_{i j}}+\frac{1}{\mu_{e j}}+\frac{1}{\mu_{j e}}
\end{aligned}
$$

Here, $\mu_{\mathrm{ei}}$ is the transmission service rate from the IoT device e to $\mathrm{FN} i, \mu_{\mathrm{ie}}$ is the transmission service rate from the $\mathrm{FN} i$ to the IoT device e, $\mu_{\mathrm{je}}$ is the transmission service rate from neighbouring FN j to e, $\mu_{\mathrm{ej}}$ is the transmission service rate from the IoT device e to node $j, \mu_{i j}$ is the transmission service rate from the $F N i$ to the node $j, T_{e i}$ is the $T_{D}$ from IoT device e to the $\mathrm{FN}$ i, $\mathrm{T}_{\text {ie }}$ is the $\mathrm{T}_{\mathrm{D}}$ from the $\mathrm{FN}$ i to IoT device $e, T_{e j}$ is the $T_{D}$ from the IoT device e to the neighbouring $F N j, T_{j e}$ is the $T_{D}$ from the neighbouring FN $j$ to the IoT device e, $T_{j i}$ is the $T_{D}$ from the neighbouring $\mathrm{FN} j$ to the $\mathrm{FN} i, \mathrm{~T}_{\mathrm{ij}}$ is the $\mathrm{T}_{\mathrm{D}}$ from the $\mathrm{FN} i$ to neighbouring $\mathrm{FN} \mathrm{j}, g_{e i}, g_{i e}, g_{j e}, g_{e j}, g_{i j}$ and $g_{j i}$ are the channel gains for $\mu_{\mathrm{ei}}, \mu_{\mathrm{ie}}, \mu_{\mathrm{je}}, \mu_{\mathrm{ej}}, \mu_{\mathrm{ij}}$, and $\mu_{\mathrm{ji}}, \mathrm{B}_{\mathrm{e}}, \mathrm{B}_{\mathrm{i}}$, and $\mathrm{B}_{\mathrm{j}}$ are the bandwidth for the IoT device e , for node $i$, and for node $j, \gamma_{1}, \gamma_{3}, \gamma_{5}, \gamma_{7}, \gamma_{9}$, and $\gamma_{11}$ are the path loss exponent, $\gamma_{2}, \gamma_{4}, \gamma_{6}, \gamma_{8}, \gamma_{10}$, and $\gamma_{12}$ are the Path loss constant, $P_{t x, e}, P_{t x, i}$ and $P_{t x, j}$ are the transmission power for node e, node $\mathrm{i}$, and node $\mathrm{j}, \mathrm{d}_{\mathrm{e}}$, $\mathrm{d}_{\mathrm{ie}}, \mathrm{d}_{\mathrm{ej}}, \mathrm{d}_{\mathrm{je}}, \mathrm{d}_{\mathrm{ij}}$, and $\mathrm{d}_{\mathrm{ji}}$ are the distance between e and $\mathrm{i}, \mathrm{i}$ and $\mathrm{e}, \mathrm{e}$ and $\mathrm{j}, \mathrm{j}$ and e, i and j, and j and i, $N_{o}^{e}, N_{o}^{i}$, and $N_{o}^{j}$ are the noise densities from nodes e to $i$ and $j, i$ to $j$ and e, and $j$ to $i$ and $e, \lambda_{e i}$ and $\lambda_{\text {ie }}$ are arrival rates of task data from node e to node $i$, and from node i to node e, $\lambda_{e j}$ and $\lambda_{\mathrm{je}}$ are the arrival rate of task data from node e to node $j$, and from node $j$ to node e, $\lambda_{\mathrm{ij}}$ and $\lambda_{\mathrm{ji}}$ are the arrival rate of task data from node $\mathrm{i}$ to node $\mathrm{j}$, and from node $\mathrm{j}$ to node $\mathrm{i}$

Network Delay $\left(\mathrm{N}_{\mathrm{D}}\right)$ : Networks delay $\left(\mathrm{N}_{\mathrm{D}}\right)$ incurred the delay which depends upon the total number of packets from the sensor network to fog network and fog network to sensor network. Network delay depends upon every hop delay as well as total packet sent from the end-user node e to FN i, FN i to neighboring node j, and from FN $\mathrm{j}$ to end-user node e and also assuming that there is equal latency for each hop delay. The network delay is calculated as:

$$
\begin{aligned}
& \mathrm{N}_{\mathrm{D}}=\mathrm{N}_{\mathrm{D}} \text { from node e to } \mathrm{i}+\mathrm{N}_{\mathrm{D}} \text { from node } \mathrm{i} \text { to } \mathrm{j}+\mathrm{N}_{\mathrm{D}} \text { from node } \\
& \mathrm{j} \text { to e } \\
& \quad=\frac{d_{\propto} h_{c} e}{X_{P}}+\frac{d_{\propto} h_{c} i}{X_{P}}+\frac{d_{\propto} h_{c} j}{X_{P}}=\frac{d_{\propto} h_{c}}{X_{P}}(e+i+j)
\end{aligned}
$$

Where $h_{c}$ and $d_{\alpha}$ are the hop count and hop delay.

Computation Delay $\left(\mathrm{C}_{\mathrm{D}}\right)$ : When task computation is done by $\mathrm{FN}$, there exists a waiting queue in the task computation queue due to the prior task available in the queue for processing. The neighbouring FNs are not just receiving the task from a single source node they receive it from multiple nodes and also from end-users. Hence, the computation queue can be computed as an $\mathrm{M} / \mathrm{D} / 1$ system, neglecting the loss of packets, with the task data arrival rate and the computation latency of 
FNs that can be expressed as

$$
\begin{aligned}
& \mathrm{C}_{\mathrm{ei}}=\frac{\lambda_{\mathrm{ei}}}{2 \mu_{i}\left(\mu_{i}-\lambda_{e i}\right)}+\frac{1}{\mu_{i}}+\frac{\lambda_{\mathrm{ei}}}{C_{s}} \\
& \mathrm{C}_{\mathrm{ij}}=\frac{\lambda_{\mathrm{ij}}}{2 \mu_{j}\left(\mu_{j}-\lambda_{i j}\right)}+\frac{1}{\mu_{j}}+\frac{\lambda_{\mathrm{ij}}}{C_{s}^{\prime}}
\end{aligned}
$$

Total computation delay can be calculated as

$$
\begin{aligned}
& \mathrm{C}_{\mathrm{D}}=\mathrm{C}_{\mathrm{ei}}+\mathrm{C}_{\mathrm{ij}}=\frac{\lambda_{\mathrm{ei}}}{2 \mu_{i}\left(\mu_{i}-\lambda_{e i}\right)}+\frac{1}{\mu_{i}}+\frac{\lambda_{\mathrm{ei}}}{C_{S}}+ \\
& \frac{\lambda_{\mathrm{ij}}}{2 \mu_{j}\left(\mu_{j}-\lambda_{i j}\right)}+\frac{1}{\mu_{j}}+\frac{\lambda_{\mathrm{ij}}}{C_{s}^{\prime}} \\
& =\frac{\lambda_{\mathrm{ei}}}{2 \mu_{i}\left(\mu_{i}-\lambda_{e i}\right)}+\frac{\lambda_{\mathrm{ij}}}{2 \mu_{j}\left(\mu_{j}-\lambda_{i j}\right)}+\frac{1}{\mu_{i}}+\frac{1}{\mu_{j}}+\frac{\lambda_{\mathrm{ei}}}{C_{S}}+\frac{\lambda_{\mathrm{ij}}}{C_{S}^{\prime}}
\end{aligned}
$$

Where $\mu_{i}$ and $\mu_{j}$ are the hardware parameter at node $\mathrm{i}$ and node $\mathrm{j}$, $C_{s}$ and $C_{s}{ }^{\prime}$ are the speeds of CPU at node $\mathrm{i}$ and node $\mathrm{j}$.

Here, the first term used as a waiting time in the computation queue, and the second term is used as delay occurred for tracking the proper application used for task computation. The tracking delay depends upon the quality of the hardware used.

The total latency $\left(\mathrm{T}_{\mathrm{L}}\right)$ or total delay time can be calculated as the sum of transmission delay, network delay, and computation delay

$$
\begin{aligned}
& \mathrm{T}_{\mathrm{L}}=\mathrm{T}_{\mathrm{D}}+\mathrm{N}_{\mathrm{D}}+\mathrm{C}_{\mathrm{D}} \\
& =\frac{\lambda_{\mathrm{ei}}}{2 \mu_{e i}\left(\mu_{e i}-\lambda_{e i}\right)}+\frac{\lambda_{\mathrm{ie}}}{2 \mu_{i e}\left(\mu_{i e}-\lambda_{i e}\right)}+\frac{\lambda_{\mathrm{ij}}}{2 \mu_{i j}\left(\mu_{i j}-\lambda_{i j}\right)}+ \\
& \frac{\lambda_{\mathrm{ji}}}{2 \mu_{j i}\left(\mu_{j i}-\lambda_{j i}\right)}+\frac{\lambda_{\mathrm{ej}}}{2 \mu_{e j}\left(\mu_{e j}-\lambda_{e j}\right)}+ \\
& \frac{\lambda_{\mathrm{je}}}{2 \mu_{j e}\left(\mu_{j e}-\lambda_{j e}\right)}+\frac{1}{\mu_{e i}}+\frac{1}{\mu_{i e}}+\frac{1}{\mu_{j i}}+\frac{1}{\mu_{i j}}+\frac{1}{\mu_{e j}}+ \\
& \frac{1}{\mu_{j e}}+\frac{h_{c}}{X_{P}}\left(d_{e} e+d_{i} i+d_{j} j\right)+\frac{\lambda_{\mathrm{ei}}}{2 \mu_{i}\left(\mu_{i}-\lambda_{e i}\right)}+\frac{\lambda_{\mathrm{ij}}}{2 \mu_{j}\left(\mu_{j}-\lambda_{i j}\right)}+ \\
& \frac{1}{\mu_{i}}+\frac{1}{\mu_{j}}+\frac{\lambda_{\mathrm{ei}}}{C_{S}}+\frac{\lambda_{\mathrm{ij}}}{C_{s}^{\prime}}
\end{aligned}
$$

\section{Results AND Discussion}

In this section, we discussed the performance of the model. In this model, data is transferred from one layer to another layer started from IoT devices and reaches to cloud through a fog environment. The time consumed by data in travelling is calculated. As data is classified, it is processed and as per requirement, the data is sent to the end-user or cloud. To complete the research task, we use the tool of python editor. The result will be visualized after the completion of the simulation process. Here, the data set is divided into tenfold as we applied a $\mathrm{k}$-fold random forest learning algorithm. $70 \%$ of the data set will use for training purposes whereas $30 \%$ of data used for testing purposes. Python 3.7 is used as a platform for implementing this work. Random forest algorithm classifies the data in high risk, low risk, and normal with the accuracy of $92 \%$ in the proposed work. It has taken 14 seconds as computation time.

For the simulation, we performed several tests for monitoring devices with five different configurations. The evaluation of latency, usage of the network, and consumption of RAM were performed by the simulations. The ifogsim [11] simulator is used to simulate the fog network and nodes. The evaluation of the transmission delay, computation delay, and network delay is simulated through the ifogsim [11] simulator. This simulator creates the physical topologies and they are programmed with Java API. JSON file format is used to store the updated and modified topologies. By varying the size of topology, the performance of simulation is evaluated.

The FNs are swapping the data packets among the system entities during the simulation. The wi-fi connection is established between Fog and IoT devices. In the process of testing the performance, different topologies parameters are used concerning the different number of fog and IoT devices. IoT-sensor, FNs, and cloud data centers as servers are used as physical topology parameters in the simulating tool. By varying the size of topology, the performance of simulation was evaluated.

All configurations (number five) such as configure.1, configure.2, configure.3, configure.4, and configure.5 are simulated with physical topologies on the simulated tool. This system is generated for the performance analysis of proposed work in the fog computing environment. The IoT_sensor device has 1200 million instructions as a CPU length, a network-length of 21000 bytes, and inter-arrival time (average) at data packet arrival of $20 \mathrm{~ms}$.

The details of the used fog device, IoT-sensor, and link of the network are shown in Table VII and Table VIII.

TABLE VII. Details of Fog Device Parameters

\begin{tabular}{|c|c|c|}
\hline Type of device & $\begin{array}{c}\text { Processing speed } \\
\text { (G.Hz) }\end{array}$ & Ram (MegaBytes) \\
\hline Fog-device & 2.60 & 2.0 \\
\hline Cloud-server & 4.0 & 4.0 \\
\hline
\end{tabular}

TABLE VIII. Details of Network Link Parameters

\begin{tabular}{|c|c|c|}
\hline Source node & Destination node & Latency $(\mathbf{m s})$ \\
\hline IoT_sensor1 & Fog-device & 45.0 \\
\hline IoT_sensor2 & Fog-device & 45.0 \\
\hline IoT_sensor3 & Fog-device & 50.0 \\
\hline Fog-device & Cloud-server & 75.0 \\
\hline
\end{tabular}

A comparison in transmission delay between fog and cloud environment is shown in Fig. 3. First of all, a link (tuple) is generated by IoT-sensors, and the connection is established with available routers, gateways, and connected FNs. After getting the data packet on fog servers, processing and distributing to other neighbouring FNs, then data packets are received by the end-user.

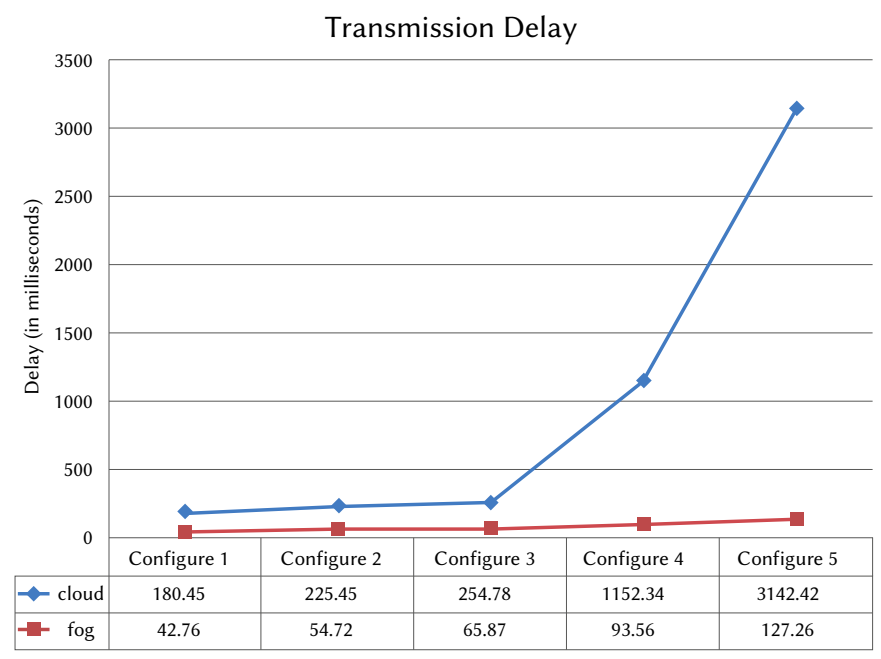

Fig. 3. Fog computing and cloud computing comparison for $T_{D}$.

The comparison of network delay between fog and cloud computing is shown in Fig. 4. When the transmission of data occurs between 
IoT-sensors and fog servers, the hop counts decreases. Fig. 4. shows the reduction in network latency. When there is a large volume of data transmitted between IoT-sensors and cloud servers, there is an important increase in network latency for the cloud network while this is kept low for the fog network.

The comparison between fog and cloud computing computation delay is shown in Fig. 5. When task data reaches to FNs, it starts computing the data, and the computation depends upon the parameters such as the speed of the processing unit, hardware performance, and size of the data packet.

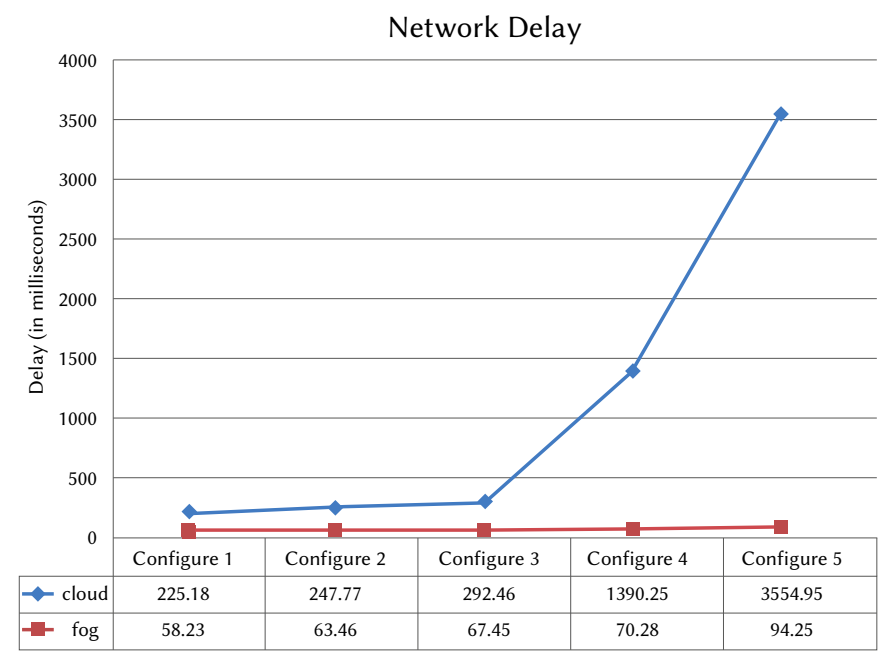

Fig. 4. Fog computing and cloud computing comparison for $\mathrm{N}_{\mathrm{D}}$.

Fig. 6 shows the consumption of usage of networks in fog and cloud computing environments. FNs are deployed over certain regions to overcome network congestion.

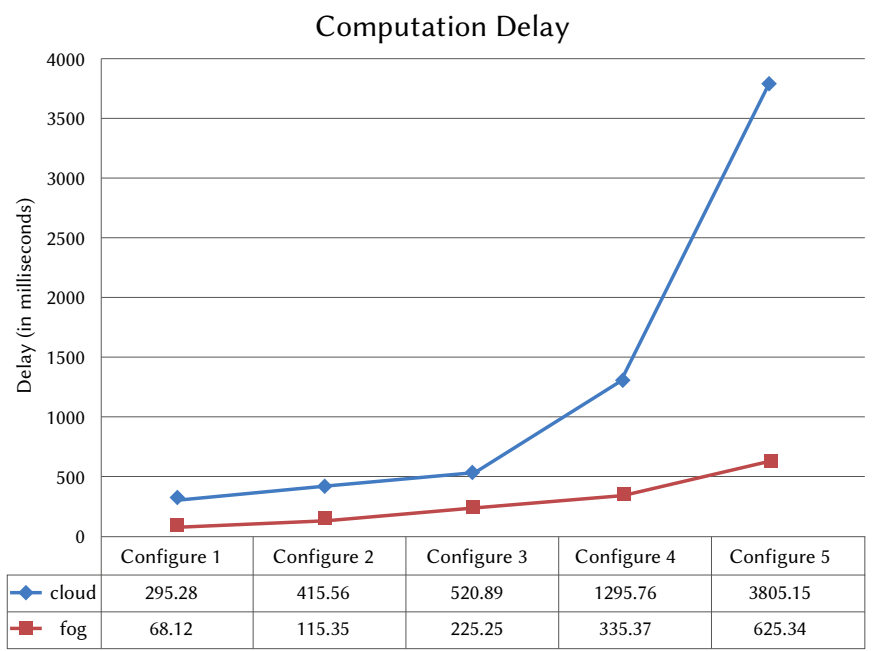

Fig. 5. Fog computing and cloud computing comparison for $C_{D}$.

In these simulation results, the various physical topology configurations are used in the fog computing environment. As a result, the average result of transmission delay is $76.834 \mathrm{~ms}$, the average result of network delay is $70.734 \mathrm{~ms}$, and $273.886 \mathrm{~ms}$ for average computation delay. The usage of the network is also minimized with the average result is kilo bytes. The existing state-of-art is compared with the proposed algorithm that minimized latency by $94-95 \%$. We compared the proposed model by Hermes [23], iFogStor [18], and Hipster [16], where an improvement in the minimization of latency is by $16 \%$ with the model presented by Hermes, an $86 \%$ reduction in latency is demonstrated as a comparison to cloud computing by iFogStor, and Hipster improves the latency for web-searching by $80-90 \%$. Raafat [32] shows the reduction in overall service latency by $21.9 \%$ to $46.6 \%$ in the fog environment.

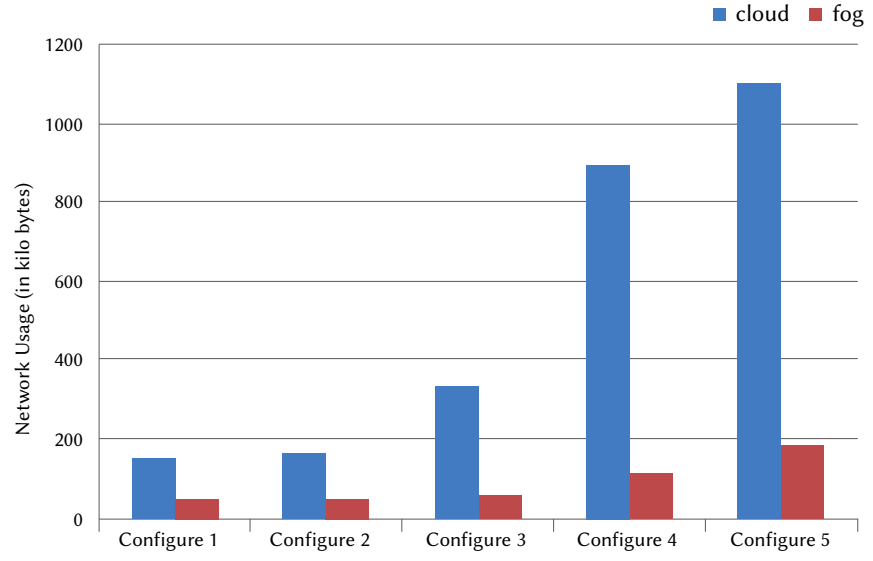

Fig. 6. Fog computing and cloud computing comparison for Network Usage.

\section{CONCLUSION}

Classification of health data and minimization of latency is the most challenging task in e-healthcare, where the fog server is receiving a high volume of task data. Due to the complicated nature of data, fog computing technology becomes essential and important to minimize latency in e-healthcare. In this paper, we presented a novel intelligent multimedia data segregation (IMDS) scheme using machine learning ( $\mathrm{k}$-fold random forest) in the fog computing environment. The latency parameters such as transmission delay, network delay, and computation delay are evaluated and it shows the reduction in the high latency. The proposed model is improving the quality of service in e-healthcare and suitable for heterogeneous networks. The latency and usage of the network is a part of QoS. Hence, minimizing the latency and usage of network improves the QoS. In the future, the quality of services in e-healthcare and latency for high-risk data can be improved by using $5 \mathrm{G}$ as higher internet connectivity. A smart healthcare system can be implemented in a different hospital through the fog model.

\section{ACKNOWLEDGMENT}

The authors would like to thanks to Department of Computer Science \& Information Technology, Sam Higginbottom University of Agriculture, Technology and Sciences (SHUATS), Allahabad, U.P., India to give this platform to work. Thanks to all my seniors in the department.

Conflicts of interest/Competing interests - There are no conflicts of interests.

\section{REFERENCES}

[1] C. S. Nandyala and H. K. Kim, "From cloud to fog and IoT-based real-time U-healthcare monitoring for smart homes and hospitals", International fournal of Smart Home, vol. 10, no. 2, 2016, pp. 187-196.

[2] S. Cirani, G. Ferrari, N. Iotti, M. Picone M, "The IoT hub: A fog node for seamless management of heterogeneous connected smart objects", 12th Annual IEEE International Conference on Sensing, Communication, and Networking-Workshops (SECON Workshops), 2015.

[3] L.M. Vaquero, L. Rodero-Merino, "Finding your way in the fog: Towards a comprehensive deffinition of fog computing", ACM SIGCOMM 
Computer Commun. Review, vol. 44, no. 5, 2014, pp. 27-32.

[4] F. Bonomi, R. Milito, J. Zhu, S. Addepalli S, "Fog computing and its role in the internet of things", in Proceedings of the First Edition of the MCC Workshop on Mobile Cloud Computing, 2012, pp. 13-16.

[5] S. Yi, C. Li C, Q. Li Q, "A survey of fog computing: Concepts, applications and issues", in Proceedings of the 2 Workshop on Mobile Big Data, ACM, 2015, pp. 37-42.

[6] P. G. Lopez, A. Montresor, D. Epema, A. Datta, T. Higashino, A. Iamnitchi, M. Barcellos, P. Felber, E. Riviere, "Edge-centric computing: Vision and challenges", SIGCOMM Computer Communication Review, vol. 45, no. 5, 2015, pp. 37-42.

[7] M. Chiang and T. Zhang, "Fog and IoT: An overview of research opportunities", IEEE Internet of Things fournal, vol. 3, no. 6, 2016, pp. 854864.

[8] T. N. Gia, M. Jiang, A. M. Rahmani, T. Westerlund, P. Liljeberg, H. Tenhunen, "Fog computing in healthcare internet of things: A case study on ecg feature extraction", in 2015 IEEE international conference on computer and information technology; ubiquitous computing and communications; dependable, autonomic and secure computing; pervasive intelligence and computing, IEEE,, 2015, pp. 356-363.

[9] S.C. Hung, D. Liau, S.Y. Lien, K.C. Chen, "Low latency communication for Internet of Things", in IEEE/CIC International Conference on Communications in China (ICCC), IEEE, 2015, pp. 1-6.

[10] G. Lee, W. Saad, M. Bennis, "An online optimization framework for distributed fog network formation with minimal latency", IEEE Transactions on Wireless Communications, vol. 18, no. 4, 2019, pp. 2244-2258.

[11] H. Gupta, A. V. Dastjerdi, S. K. Ghosh, R. Buyya, "iFogSim: A toolkit for modeling and simulation of resource management techniques in the Internet of Things, Edge and Fog computing environments", Practice and Experience, vol. 47, no. 9, 2017, pp.1275-1296.

[12] L. Skorin-Kapov and M. Matijasevic, "Analysis of QoS requirements for e-health services and mapping to evolved packet system QoS classes", International journal of telemedicine and applications. 2010.

[13] J. R. Gallego, A. Hernandez-Solana, M. Canales, J. Lafuente, A. Valdovinos, J. Fernandez-Navajas, "Performance analysis of multiplexed medical data transmission for mobile emergency care over the UMTS channel", IEEE transactions on information technology in biomedicine. Vol. 9, no. 1, 2005, pp.13-22.

[14] C. A. Silva, G. S. Aquino, S.R.M. Melo, D. J. B. Egidio, "A Fog ComputingBased Architecture for Medical Records Management", Wireless Communications and Mobile Computing, 2019.

[15] M. G. R. Alam, Y. K. Tun, C. S. Hong, "Multi-agent and reinforcement learning based code offloading in mobile fog", in International Conference on Information Networking (ICOIN), IEEE, 2016, pp. 285-290.

[16] R. Nishtala, P. Carpenter, V. Petrucci, X. Martorell, "Hipster: Hybrid task manager for latency-critical cloud workloads", in IEEE International Symposium on High Performance Computer Architecture (HPCA), IEEE 2017, pp. 409-420.

[17] T. N. Gia, M. Jiang, V. K. Sarkar, A. M. Rahmani, T. Westerlund, P. Liljeberg, H. Tenhunen H, "Low-cost fog-assisted healthcare IoT system with energy-efficient sensor nodes" in Proceedings of 13th international wireless communications and mobile computing conference (IWCMC), IEEE, 2017, pp. 1765-1770.

[18] M. I. Naas, P. R. Parvedy, J. Boukhobza, L. Lemarchand, "iFogStor: an IoT data placement strategy for fog infrastructure", in IEEE 1st International Conference on Fog and Edge Computing (ICFEC), 2017, pp. 97-104.

[19] A. M. Rahmani, T. N. Gia, B. Negash, A. A. I. Azimi, M. Jiang, P. Liljeberg, “" Exploiting smart e-Health gateways at the edge of healthcare Internetof-Things: A fog computing approach", Future Generation Computer Systems, vol. 78, 2018, pp. 641-658.

[20] J. Wu, M. Dong, K. Ota, J. Li, Z. Guan, "FCSS: Fog computing based content-aware filtering for security services in information centric social networks", IEEE Transactions on Emerging Topics in computing, 2017.

[21] A. Brogi and S. Forti, "QoS-aware deployment of IoT applications through the fog", IEEE Internet of Things Journal, vol. 4, no. 5, 2017, pp.1185-1192.

[22] A. Shahzad, Y. S. Lee, M. Lee, Y. G. Kim, N. Xiong, "Real-Time CloudBased Health Tracking and Monitoring System in Designed Boundary for Cardiology Patients", Journal of Sensors, 2018.

[23] Y. H. Kao, B. Krishnamachari, M. R. Ra, F. Bai, "Hermes: Latency optimal task assignment for resource constrained mobile computing". IEEE
Transactions on Mobile Computing, vol. 16, no. 11, 2017, pp. 3056-3069.

[24] G. Li, J. Wu, J. Li, K. Wang, T. Ye, "Service popularity-based smart resources partitioning for fog computing-enabled industrial Internet of Things", IEEE Transactions on Industrial Informatics, vol. 14, no. 10, 2018, pp.:4702-4711.

[25] R. Mahmud, F. L. Koch, R. Buyya, "Cloud-fog interoperability in IoTenabled healthcare solutions", in Proceedings of the 19th international conference on distributed computing and networking, 2018, pp. 1-10.

[26] N. T. Dinh and Y. Kim, "An Efficient Availability Guaranteed Deployment Scheme for IoT Service Chains over Fog-Core Cloud Networks", Sensors. 18(11):3970, 2018.

[27] M. M. Ahsan, I. Ali, M. Imran, M. Y. I. Idris, S. Khan, A. Khan, "A Fogcentric Secure Cloud Storage Scheme", IEEE Transactions on Sustainable Computing, 2019.

[28] H. Rafique, M. A. Shah, S. U. Islam, T. Maqsood, S. Khan, C. Maple, "A Novel Bio-Inspired Hybrid Algorithm (NBIHA) for Efficient Resource Management in Fog Computing", IEEE Access, vol. 7, 2019, pp. 115760115773.

[29] J. Li, T. Zhang, J. Jin, Y. Yang, D. Yuan, L. Gao, "Latency Estimation for Fog-based Internet of Things", in 27th International Telecommunication Networks and Applications Conference (ITNAC), IEEE, 2017, pp. 1-6. 2017.

[30] C. Thota, R. Sundarasekar, G. Manogaran, R. Varatharajan, M. K. Priyan, "Centralized fog computing security platform for IoT and cloud in healthcare system", in Fog computing: Breakthroughs in research and practice, IGI global, 2018, pp. 365-378.

[31] T. Aladwani, "Scheduling IoT Healthcare Tasks in Fog Computing Based on their Importance", in Proc of 16th International Learning \& Technology Conference, Procedia Computer Science, 2019, pp. 560-569.

[32] O. A. Raafat, A. K. Mazin, L. Taha, E. F. Khaled, "Scheduling Internet of Things Requests to Minimize Latency in Hybrid Fog-Cloud Computing", Future Generation Computer Systems, 111, 2020, pp. 539-551.

[33] J. Pan J and J. McElhannon, "Future edge cloud and edge computing for internet of things applications", IEEE Internet of Things fournal, vol. 5, no. 1, 2017. pp. 439-49.

[34] Z. Alam, M. S. Rahman, M. S. Rahman, "A Random Forest based predictor for medical data classification using feature ranking", Informatics in Medicine Unlocked, 15, 100180, 2019.

[35] C. Chakraborty, "Computational approach for chronic wound tissue characterization", Informatics in Medicine Unlocked, 17, 100162, 2019.

[36] C. Chakraborty C and R. Roy, "Markov Decision Process based Optimal Gateway Selection Algorithm" International Journal of Systems, Algorithms \& Applications (IJSAA), 2012, pp. 48-52.

[37] C. Chakraborty, R. Roy, S. Pathak, S. Chakrabarti, "An Optimal Probabilistic Traffic Engineering Scheme for Heterogeneous Networks", Int. Journal of Fuzzy Systems, vol. 3, no. 2, 2011, pp. 35-39.

[38] M. Nilashi, H. Ahmadi, A. A. Manaf, T. A. Rashid, S. Samad, L. Shahmoradi, A. Nahla, A. Elanz, "Coronary Heart Disease Diagnosis Through SelfOrganizing Map and Fuzzy Support Vector Machine with Incremental Updates", International fournal of Fuzzy Systems, 2020, pp.1-13.

[39] T.A. Rashid, S. M. Abdullah, R. M. Abdullah, "An intelligent approach for diabetes classification, prediction and description", Innovations in BioInspired Computing and Applications, Springer, Cham, 2016, pp. 323-335.

[40] H. Mahmud, M. Mohammadi,, N. Ali, T. A. R. Khan, K. A. S. Nawzad, R. M. D. Omer, L. Joan, "Technologies in medical information processing", Advances in Telemedicine for Health Monitoring, Technologies, Design and Applications, 31, 2020.

[41] T. A. Rashid, M. K. Hassan, M. Mohammadi, K. Fraser, "Improvement of variant adaptable LSTM trained with metaheuristic algorithms for healthcare analysis", Advanced Classification Techniques for Healthcare Analysis, IGI Global, 2019, pp. 111-131.

[42] J. C. W. Lin, Y. Shao, P. Fournier-Viger, F. Hamido, "BILU-NEMH: A BILU neural-encoded mention hypergraph for mention extraction", Information Sciences, 496, 2020, pp. 53-64.

[43] J. C. W. Lin, Y. Shao, J. Zhang, U. Yun, "Enhanced sequence labeling based on latent variable conditional random fields", Neurocomputing, 403, 2020, pp. 431-440.

[44] U. Ahmed, J. C. W. Lin, G. Srivastava, M. Aleem, "A load balance multischeduling model for OpenCL kernel tasks in an integrated cluster", Soft Computing, 2020, pp. 1-14.

[45] T. A. Rashid, P. Fattah, D. K. Awla, "Using accuracy measure for improving 
the training of LSTM with metaheuristic algorithms", Procedia Computer Science, 140, 2018, pp. 324-333.

[46] A. Janosi, M. Pfisterer, W. Steinbrunn, R. Detrano, J. Schmid, S. Sandhu, K. Gupta, S. Lee , V. Froelicher, UCI Machine Learning Repository 2019. https://archive.ics.uci.edu/ml/datasets/heart+disease

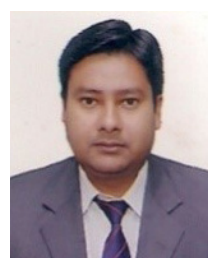

\section{Amit Kishor}

Er. Amit Kishor pursuing a Ph.D. in Computer Engineering from the Department of Computer Science and Information Technology, in the Faculty of Engineering and Technology, Sam Higginbottom University of Agriculture, Technology and Sciences, Naini, Allahabad, India. He is also working as an Assistant Professor in the Department of Computer Science and Engineering \& I.T., Subharti Institute of Engineering and Technology, Swami Vivekanand Subharti University, Meerut. India His area of interest is cloud computing, Algorithms, Artificial Intelligence, and Data Structures. He has published more than 20 papers in reputed international journals. He is also a member of an International body of International Association of Engineers (IEANG).

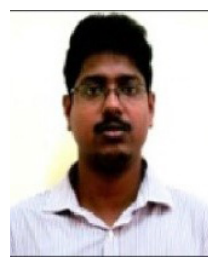

Chinmay Chakraborty

Dr. Chinmay Chakraborty is working as an Assistant Professor (Sr.) in the Dept. of Electronics and Communication Engineering, Birla Institute of Technology, Mesra, India. He worked at the Faculty of Science and Technology, ICFAI University, Agartala, Tripura, India as a Sr. lecturer. He worked as a Research Consultant in the Coal India project at Industrial Engineering \& Management, IIT Kharagpur. He worked as a project coordinator of the Telecommunication Convergence Switch project under the Indo-US joint initiative. He also worked as a Network Engineer in System Administration at MISPL, India. His main research interests include the Internet of Medical Things, Wireless Body Area Network, Wireless Networks, Telemedicine, m-Health/e-health, and Medical Imaging. Dr. Chakraborty has published sixty papers at reputed international journals, conferences, book chapters, and books. He is an Editorial Board Member in the different Journals and Conferences. He is serving as a Guest Editor of MDPI-Future Internet Journal, Internet Technology Letters, Wiley, and has conducted a session of SoCTA-19, ICICC - 2019, and also a reviewer for international journals including IEEE Access, Elsevier, Springer, Taylor \& Francis, IGI, IET, TELKOMNIKA Telecommunication Computing Electronics and Control, and Wiley. Dr. Chakraborty is co-editing Eight books on Smart IoMT, Healthcare Technology, and Sensor Data Analytics with CRC Press, IET, Pan Stanford, and Springer. He has served as a Publicity Chair member at renowned international conferences including IEEE Healthcom, IEEE SP-DLT. Dr. Chakraborty is a member of Internet Society, Machine Intelligence Research Labs, and Institute for Engineering Research and Publication. He received a Young Research Excellence Award, Global Peer Review Award, Young Faculty Award, and Outstanding Researcher Award.

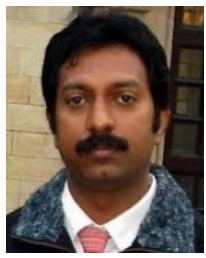

\section{Wilson Jeberson}

Prof.(Dr.) Wilson Jeberson was awarded Ph.D. degree in Computer Science and Communication, from Sam Higginbottom Institute of Agriculture, Technology \& Sciences, University, Allahabad, India. He has received the MCA in computer Application and MBA in Management from Madurai Kamaraj University Tamilnadu, India. He had worked as Programmer at National Informatics Centre (NIC), Govt. of India, from 1999 to 2000. He also worked as Senior Software Engineer cum DBA at Quintessence Technologies Limited - Trivandrum, Kerala, India, from 2000 to 2002 and as Senior System Analyst at Netcare Technologies-Trivandrum, Kerala, India from 2002 to 2003.Currently he is working as Professor \& Head, Department of Computer Science \& Information Technology in Sam Higginbottom University of Agriculture, Technology \& Sciences. Prayagraj, India from 2003. He has published more than 70 papers in reputed International journals and more than 15 Papers in National \& International Proceedings. Some of the best papers are published in Open Computer Science (Formerly: Central European Journal of Computer Science) Published by De Gruyter Open (Formerly: Springer Verlag) titled "Survey of Context Information Fusion for Ubiquitous Internet-of- Things (IoT)", International Journal Elsevier, Science Direct titled "Covert Communication Using Arithmetic Division Operation", :International Journal Elsevier, Science Direct Titled "Fortune at the bottom of the Classifier Pyramid: A novel approach to Human Activity Recognition". A research paper was selected as best one among the top 20 papers presented in the Second GMSARN International Conference at Pattaya, Thailand and was published in International Journal of AIT , Thailand titled "An approach to ICT enabled solution architecture for critical Social Security issues and challenges for e-Governance". He has coauthored two books which are published in Narosa Publications and another in Springer International. He has presented research papers in international conferences in Singapore and Thailand and also attended a training program in Galilee International institute of Management, Israel. He is also member of many national and international bodies including Computer Society of India (CSI) and International Association of Engineers (IAENG), Hong Hong. He was the Principal investigator for two funded projects one funded by Computer Society of India Education Directorate, Chennai-India and another by SHIATS. $\mathrm{He}$ is one of the editorial board members of various journals including Scientific \& Academic Publishing Co., Journal Name: Software Engineering, USA. 